\title{
Etude du profil cultural de deux sites, l'un infesté, l'autre non, par Heterodera avenae Woll.: conséquences sur le développement radiculaire du blé tendre d'hiver
}

\author{
R. Rivoal ${ }^{1}$ et J.M. Rivière 2 \\ avec la collaboration technique de P. Penard et de A. Simon \\ 1 INRA, centre de recherches de Rennes laboratoire de zoologie, BP 2935630 Le Rheu; \\ 2 ENSA, chaire de sciences du sol, 65 rue de Saint-Brieuc, 35000 Rennes, France \\ (reçu le 6 novembre 1988, accepté le 18 avril 1989)
}

Résumé - Le nématode à kystes Heterodera avenae Woll., est un parasite obligatoire des céréales. Son action a été étudiée sur le système radiculaire du blé, dans un sol calcaire de la région d'Argentan (Orne). Pour 2 sites expérimentaux, l'un "assaini", l'autre «infestée par le nématode, avec sols parfaitement comparables, en particulier au niveau de la macroporosité, le système radiculaire du blé est significativement plus important, et aussi nettement plus superficiel, dans le second site que dans le premier.

Un sol favorable au développement des racines peut donc s'avérer non exploitable par le système radiculaire du blé, si une attaque par le parasite modifie sa croissance et son exploration naturelles. En site «infesté", la très forte densité de nématodes, localisés essentiellement dans la tranche labourée du sol, constitue un réel frein à la progression en profondeur du système radiculaire. Elle fait craindre que les racines soient encore plus superficielles pour les cultures suivantes de céréales.

profil cultural - infestation - Heterodera avenae - développement radiculaire - blé tendre d'hiver

\begin{abstract}
Summary - Study of the cultural profile in two sites infested or not infested by Heterodera avenae Woll. : consequences on the root development of the winter wheat. The cereal cyst nematode Heterodera avenae is a strict parasite to cereals. Its effect was studied on the development of the root system of the wheat in a calcareous soil near Argentan (Normandy). In two closed experimental plots "uninfested" or "infested" by the nematode, whose soil were perfectly identical, particularly for the macroporosity, the root system of the wheat was significantly more important and also clearly more superficial in the second than in the first site.

A suitable soil for the root development could be left unused by the root system of the wheat if an attack of the pathogen changed its natural growth. In an "infested" site, the very high density of nematodes, located essentially in the ploughed part of the soil, constituted a real difficulty for the exploration in depth by the roots. It would contribute also to a more superficial location of the roots for the following cereals crops.
\end{abstract}

cultural profile - infestation - Heterodera avenae - root development - winter wheat

\section{INTRODUCTION}

Le nématode à kystes Heterodera avenae Woll., parasite obligatoire des graminées, est un sérieux agent pathogène pour les cultures céréalières dans le monde entier (Meagher, 1977; Ritter, 1982). En France, cette espèce est inféodée à toutes les céréales à paille et au maïs; cependant, la gravité de ses attaques varie selon la situation géographique (Rivoal, 1973).
Ces différences résultent, en particulier, de la coïncidence entre la période d'activité du parasite et les stades phénologiques les plus sensibles des cultures (Rivoal, 1982). En situation méridionale, les dégâts sont observés essentiellement sur les céréales (blé dur et blé tendre) implantées dans le courant de l'automne, en raison de l'activité hivernale des populations locales (Rivoal et Sarr, 1983). Dans la zone septentrionale, comme dans d'autres pays d'Europe du 
Nord, les attaques du ravageur sont les plus préjudiciables aux céréales de printemps (avoine, orge, maïs), semées en pleine période d'activité du nématode (Rivoal, 1986b; Rivoal et al., 1986).

Plusieurs expérimentations ont cependant montré que le blé d'hiver n'est pas indemne des attaques du nématode dans les zones septentrionales de culture (Rivoal et al., 1986; Rivoal et Sarr, 1987). Des investigations complémentaires ont été entreprises pour préciser l'influence de $H$. avenae sur cette céréale. Elles font intervenir un dispositif expérimental où la succession, pendant 3 années, de cultivars d'Avena sativa L., résistants ou favorables au développement du ravageur, a pour but de constituer un différentiel d'infestation entre 2 sites, dont l'un est dit «assaini» et l'autre «infesté». L'avoine a été choisie pour éviter le développement du Piétin échaudage, Gaeumannomyces graminis (Sacc.) Arx et Olivier var. tritici (Walker), autre agent pathogène sérieux des cultures céréalières intensives (Turner, 1953; Lucas et al., 1986).

Cette première publication rend compte des caractéristiques pédologiques des 2 sites expérimentaux, du développement du système radiculaire d'une culture consécutive de Triticum aestivum $\mathrm{L}$. et de la distribution des populations du ravageur dans chacun des 2 profils culturaux.

\section{MATÉRIEL ET MÉTHODES}

A Argentan (Orne), 2 bandes parallèles et contiguës de $360 \mathrm{~m}^{2}(6 \times 60 \mathrm{~m})$ sont cultivées pendant 3 années consécutives, de 1983 à 1985, en avoine, soit avec le cultivar "Panema", résistant au développement de $H$. avenae, soit avec le cultivar "Peniarth", favorable à sa multiplication. Ces 2 cultivars sont menés selon les mêmes itinéraires techniques, proches de la pratique agricole locale. En octobre 1985, chaque bande est subdivisée en 5 blocs de 4 parcelles, ensemencées avec le blé tendre d'hiver cultivar «Fidel», et 3 lignées de blé issues d'un programme de sélection de variétés résistantes à partir du géniteur "Loros" (Doussinault et al., 1986).

Dans une des parcelles élémentaires de chacun des 2 sites, "assaini» et “infesté», cultivée avec le blé d'hiver cultivar "Fidel», nous avons creusé une fosse de $1,30 \mathrm{~m}$ de longueur, $1,00 \mathrm{~m}$ de largeur et $0,90 \mathrm{~m}$ de profondeur. Par suite de diverses contraintes, les études sur le profil cultural ont été effectuées à 3 dates différentes:

- le 23 juin 1986, à la floraison du blé, pour évaluer le développement de son système radiculaire et les densités de nématodes anciennement ou nouvellement formés dans le sol, ou (et) sur les racines;

- le 8 octobre 1986, après déchaumage, après réouverture et rafraichissement de la fosse sur une épaisseur d'environ $15 \mathrm{~cm}$ de ses parois, afin de procéder à une analyse pédologique, tant au niveau de la morphologie des profils (Hénin et al., 1969), que des caractéristiques physico-chimiques des sols;
- le 17 octobre 1986, en site “infesté", après l'ouverture d'une nouvelle fosse, sur un profil d'une profondeur de $50 \mathrm{~cm}$, pour tester l'influence du labour sur la distribution des nématodes.

Les études sur le développement radiculaire et sur les effectifs de nématodes concernent les 4 niveaux: $0-20 \mathrm{~cm} ; 20-40 \mathrm{~cm} ; 40-60 \mathrm{~cm} ; 60-80 \mathrm{~cm}$. Pour chaque tranche, il est prélevé 4 à $5 \mathrm{~kg}$ de terre, que l'on tamise à $5 \mathrm{~mm}$, ultérieurement, au laboratoire. Les analyses sont effectuées sur 5 échantillons de $210 \mathrm{ml}$, constitués de parties aliquotes; elles visent à déterminer les poids de racines et les densités de kystes ou de femelles blanches. Les racines sont récupérées par un tri quasi exhaustif, effectué à la pince et à l'œil nu dans le sol échantillonné. Les racines sont ensuite pesées à l'état frais.

Les kystes antérieurs à la culture du blé "Fidel" sont extraits par l'appareil de Kort; ils sont comptés puis écrasés pour établir leur contenu larvaire (Rivoal et Sarr, 1987). Les nématodes adultes nouvellement formés aux stades femelle blanche ou kyste, reconnaissables par leur revêtement sub-cristallin, sont récupérés soit par extraction du sol, en même temps que les kystes anciens, soit par broyage des racines, suivi d'une centrifugation dans une solution sucrée de densité 1, 23 (Rivoal et al. 1978).

L'échantillonnage pédologique du 8 octobre a été effectué sur les 4 horizons $0-30 \mathrm{~cm} ; 30-45 \mathrm{~cm}, 45-60$ $\mathrm{cm}, 60-80 \mathrm{~cm}$. Le même jour, l'homogénéité pédologique des 2 parcelles a été vérifiée par sondages à la tarière, jusqu'à au moins $80 \mathrm{~cm}$ de profondeur. Les analyses physico-chimiques classiques ont été réalisées au laboratoire INRA d'Analyses des sols d'Arras, alors que les analyses de pF proviennent du Laboratoire INRA de Sciences du Sol de Rennes.

L'analyse statistique des densités de racines et de nématodes dans les différents niveaux a fait intervenir une analyse de variance à 2 facteurs, suivie d'une comparaison entre les 2 situations “infestée» et "assainie", par le test multilocal (Hervé, 1982). Les densités de nématodes $(x)$ sont transformées en $\log (x+1)$, pour homogénéiser les variances. Les plus petites différences significatives (P.P.D.S.) sont fournies à $P=0,05$

\section{RÉSULTATS}

\section{Développement du système radiculaire}

Tous niveaux confondus, le système radiculaire du blé "Fidel» est beaucoup plus important dans le site "infesté", où la masse est le double de celle enregistrée en zone "assainie". Dans ce site, les racines sont réparties de manière homogène entre 0 et $60 \mathrm{~cm}$ de profondeur; on en note même dans la tranche la plus profonde (Tableau I). Dans la zone «infestée», l'essentiel des racines est localisé entre 0 et $40 \mathrm{~cm}$ de profondeur. Des traces sont observées entre 40 et $60 \mathrm{~cm}$, et il n'y a pas d'exploration entre 60 et 80 $\mathrm{cm}$. La comparaison entre sites met en évidence une différence significative des poids de racines pour les 3 niveaux compris entre 0 et $60 \mathrm{~cm}$ de profondeur. 
Tableau 1. Développement et distribution du système radiculaire du blé tendre d'hiver "Fidel» cultivé en site "assaini" ou “infesté" par Heterodera avenae; différences significatives (S) et P.P.D.S. $(\Delta)$ à $P=0,05$; les moyennes suivies de la même lettre ne sont pas significativement différentes les unes des autres.

\begin{tabular}{|c|c|c|c|c|c|c|c|}
\hline & \multirow[t]{2}{*}{ Site } & \multicolumn{4}{|c|}{ Profondeur $(\mathrm{cm})$ des niveaux de sols } & \multirow{2}{*}{$\begin{array}{l}\text { Niveaux } \\
\text { confondus }\end{array}$} & \multirow{2}{*}{$\begin{array}{c}\text { Différence } \\
\text { entre niveaux } \\
\text { "assaini/infesté» }\end{array}$} \\
\hline & & $0-20$ & $20-40$ & $40-60$ & $60-80$ & & \\
\hline \multirow{4}{*}{$\begin{array}{l}\text { Racines }(\mathrm{g}) / 210 \mathrm{ml} \\
\text { de sol prélevé }\end{array}$} & "assaini» & $0,22 a$ & $0,30 a$ & $0,28 a$ & $0,09 b$ & 0,22 & $0-20 \mathrm{~S}$ \\
\hline & & & & & & & $40-60 \mathrm{~S}$ \\
\hline & $\begin{array}{l}\text { «infesté»" } \\
(\Delta=0,29)\end{array}$ & $0,85 a$ & $0,73 a$ & $0,02 b$ & ob & 0,40 & \\
\hline & $\Delta$ (intersite) & & & & & 0,11 & 0.23 \\
\hline \multirow{3}{*}{$\begin{array}{l}\text { Racines }(\mathrm{g}) / 100 \mathrm{~g} \\
\text { de sol séché }\end{array}$} & $\begin{array}{l}\text { "assaini» } \\
(\Delta=0,05)\end{array}$ & $0,11 a$ & $0,12 a$ & $0,11 \mathrm{a}$ & $0,04 b$ & 0,10 & $\begin{array}{r}0-20 \mathrm{~S} \\
20-40 \mathrm{~S}\end{array}$ \\
\hline & $\begin{array}{l}\text { «infesté»" } \\
(\Delta=0,13)\end{array}$ & $0,40 \mathrm{a}$ & $0,29 a$ & $0,01 b$ & $\mathrm{Ob}$ & 0,18 & $\begin{array}{l}40-60 \mathrm{~S} \\
60-80 \mathrm{~S}\end{array}$ \\
\hline & $\Delta$ (intersite) & & & & & 0,05 & 0,09 \\
\hline
\end{tabular}

Les clichés des Figures 1 et 2 révèlent les différences importantes d'enracinement du blé dans les 2 sites expérimentaux. Les racines sont linéaires et fines dans la zone dépourvue de $H$. avenae, alors qu'elles présentent des arrêts de croissance et de multiples ramifications, caractéristiques de l'attaque du ravageur, dans la zone «infestée».

\section{Morphologie du profil pédologique en site "assaini"}

Le profil présente les caractéristiques suivantes:

- De 0 à $26 \mathrm{~cm}$, l'horizon labouré AP1 pour l'implantation du blé est limono-sableux, contenant environ $5 \%$ de graviers $(0,2$ à $2 \mathrm{~cm})$ et $10 \%$ de cailloux $(2$ à $7,5 \mathrm{~cm})$ de craie peu altérée et d'orientation quelconque.

L'effervescence à l'acide est moyenne et généralisée. La matière organique est très liée à la matière minérale. La structure polyédrique subanguleuse est très nette, de taille $15 \mathrm{~mm}$, avec une sous-structure grumeleuse de $3 \mathrm{~mm}$. La surface des mottes est poreuse, à raison de 5 pores visibles par $\mathrm{cm}^{2}$.

Les racines sont nombreuses, fines, saines, de direction quelconque, et pénétrant toute la masse de l'horizon, sauf les éléments grossiers. Les conduits de vers sont peu nombreux. L'hori- zon est meuble, friable, de couleur brun foncé (10 YR 3/3), sans taches et à limite inférieure nette, mais sans aucun lissage.

- De 26 à $30 \mathrm{~cm}$, l'horizon correspond à la profondeur maximale atteinte par un ancien labour AP2. La structure est polyédrique, de taille $10 \mathrm{~mm}$, avec seulement 1 pore très fin visible par $\mathrm{cm}^{2}$. Cet horizon peu poreux est compact et peu friable; la mesure au pénétromètre donne une résistance moyenne de $4,5 \mathrm{~kg} / \mathrm{cm}^{2}$, alors qu'elle n'est que de $2 \mathrm{~kg} / \mathrm{cm}^{2}$ dans le premier horizon. Les racines de blé sont peu nombreuses, verticales, et certaines sont déviées par les agrégats. Cet horizon peut être assimilé à une semelle de labour, bien qu'aucune motte ne soit très compacte.

- De 30 à 43/60 cm, l'horizon pédologique est en cours d'altération (B). La structure polyédrique subanguleuse de taille $1 \mathrm{~mm}$ est très nette. II y a en moyenne 20 pores $/ \mathrm{cm}^{2}$ de section d'agrégat. Des pseudomycéliums de calcite tendre sont localisés à la face inférieure des éléments grossiers. Les racines sont aussi nombreuses que dans l'horizon sus-jacent. Cet horizon globalement très poreux est meuble et très friable; sa couleur est brune (7,5 YR 4/4). La limite inférieure irrégulière, mais à transition brusque, pourrait être due à une paléocryoturbation.

- De 43/60 à $65 \mathrm{~cm}$, l'horizon présente une faible altération de la roche mère $C 1$. Des grains 


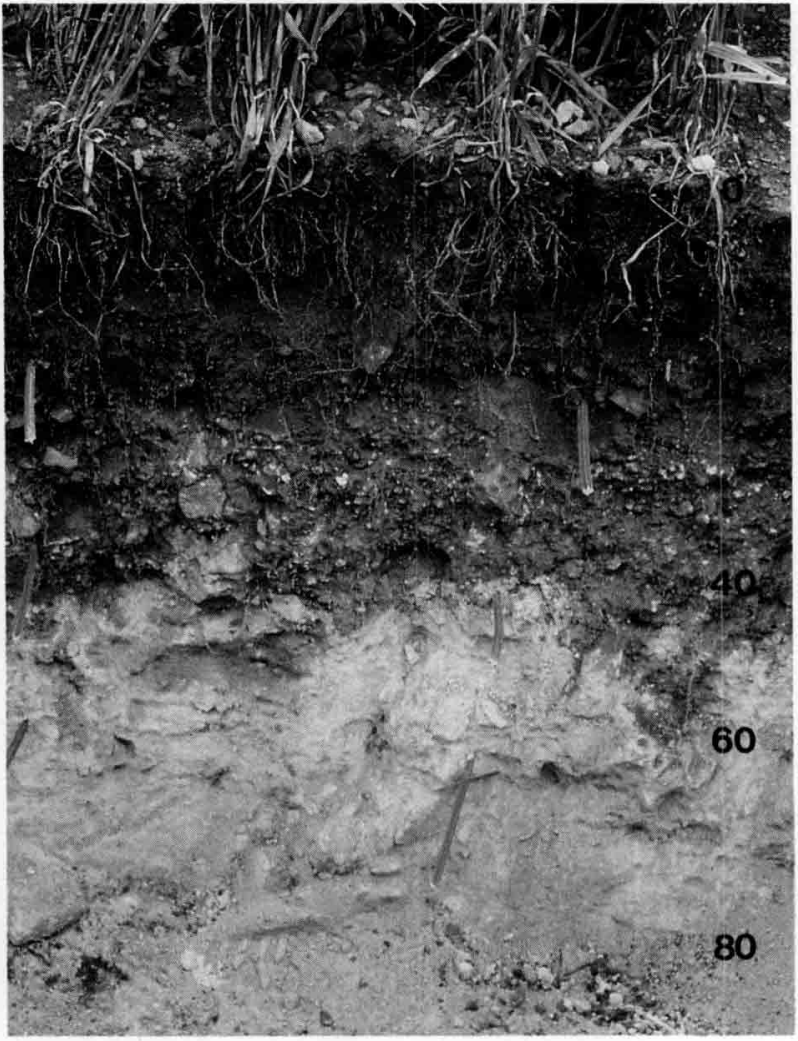

(A)

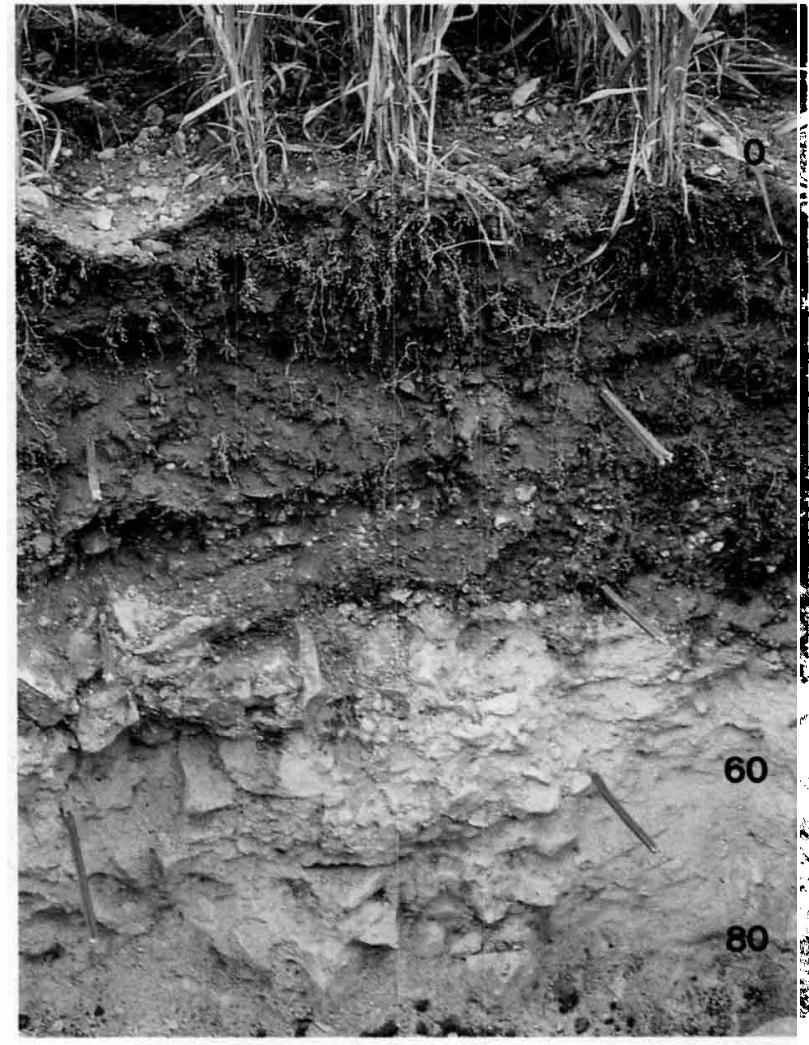

(B)

Fig. 1. Profil cultural de 2 sites expérimentaux cultivés avec le blé d'hiver «Fidel», “assaini” (A) ou «infesté" (B) par Heterodera avenae; profondeur en $\mathrm{cm}$.

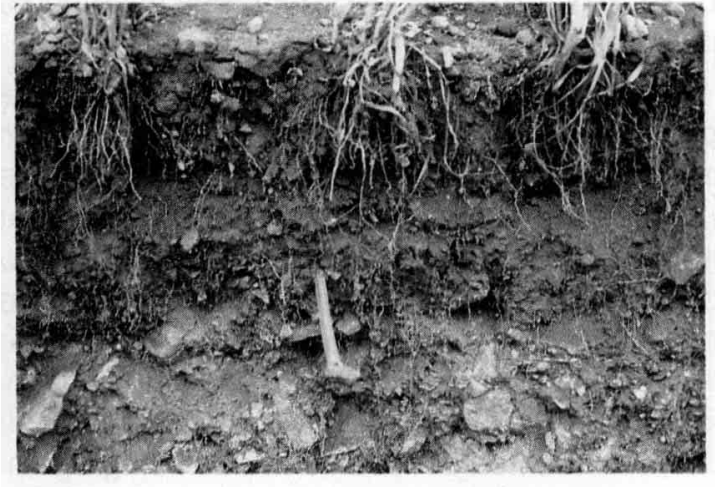

(A)

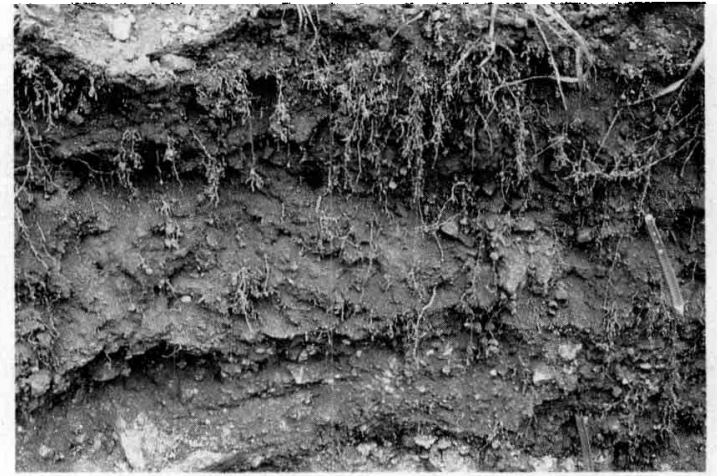

(B)

Fig. 2. Aspect des racines du blé d'hiver «Fidel» cultivé en site "assaini" (A) ou «infesté» par Heterodera avenae (B); profondeur en $\mathrm{cm}$.

pulvérulents de calcaire sont localisés sous les cailloux. La structure est continue dans la terre fine, qui est compacte avec 1 pore fin à très fin par $\mathrm{cm}^{2}$. II n'y a pas de matière organique et la couleur est brun très pâle (10 YR 7/3). De rares conduits de lombrics verticaux sont remplis de terre de l'horizon (B); ils se prolongent localement dans l'horizon sous-jacent jusqu'à $90 \mathrm{~cm}$ de profondeur et permettent l'infiltration de rares racines. Les racines, aussi nombreuses que dans l'horizon précédent, sont saines et fines; elles utilisent préférentiellement ces conduits, mais pénètrent aussi la masse de l'horizon, tout en étant déviées par les cailloux. Leur orientation est surtout verticale.

- De 65 à $100 \mathrm{~cm}$, la roche mère du sol est une craie oolithique du Bathonien moyen C2. II n'y a pas de racines dans la masse crayeuse et les grains pulvérulents de calcite enrobent toutes les faces des cailloux, dont la formation résulte vraisemblablement de la concentration de calcaire. La masse crayeuse est non altérée, peu résistante bien que compacte. Elle est très aisément friable et globalement plus poreuse que I'horizon C1. 


\section{Morphologie du profil pédologique en site «infesté»}

Ce profil est situé à $4 \mathrm{~m}$ à l'est du précédent. ॥ présente les mêmes horizons et seules les différences avec le premier profil sont reportées cidessous.

$-D e$ à $24 \mathrm{~cm}$ AP1: plus poreux, 7 pores $/ \mathrm{cm}^{2}$ au lieu de 5 .

- De 24 à $28 \mathrm{~cm}$ AP2: plus poreux, 3 pores $/ \mathrm{cm}^{2}$ au lieu de 1 et de diamètre compris entre 0 et $2 \mathrm{~mm}$ au lieu de 0 à $1 \mathrm{~mm}$. Sa compacité est plus faible, confirmée par une plus faible résistance au pénétromètre : $2,5 \mathrm{~kg} / \mathrm{cm}^{2}$ contre 4,5 dans le site "assaini». Les racines sont nombreuses, non déviées par cette semelle de labour; leur forme est, en outre, tourmentée et fortement ramifiée.

- De 28 à 35/46 (B): un peu moins poreux (16 pores $\left./ \mathrm{cm}^{2}\right)$ et un peu plus tassé $\left(3,5 \mathrm{~kg} / \mathrm{cm}^{2}\right)$ que dans la zone «assainie». La limite inférieure est seulement ondulée au lieu d'être irrégulière.

- De 35 à $58 \mathrm{~cm} \mathrm{C1:} \mathrm{l'horizon} \mathrm{est} \mathrm{plus} \mathrm{poreux}$ (2 pores $/ \mathrm{cm}^{2}$ au lieu de 1). La résistance au pénétromètre est supérieure de $0,5 \mathrm{~kg} / \mathrm{cm}^{2}$. Les racines sont très peu nombreuses et leur orientation quelconque. La couleur est plus vive (10 YR 7/4).

- De 58 à $90 \mathrm{~cm}$ C2: I'horizon ne se démarque de son homologue "assaini" que par une couleur plus vive (10 YR $7 / 4$ au lieu de 10 YR 7/3).

Ces 2 profils sont apparentés aux sols bruns calcaires et les différences de morphologie observées sont très faibles. Les porosités et les mesures de pénétrométrie ne varient que dans la zone d'imprécision classique de tous les sols cultivés, et ne font donc que manifester l'histoire culturale, sans doute un peu différente, des 2 points d'observation. II n'est pas possible objectivement de les considérer comme cause de l'enracinement nettement différent entre les 2 profils.

La teneur en éléments grossiers, estimée oculairement sur les coupes, semble présenter plus de différences d'une zone à l'autre que la morphologie générale, en particulier dans l'horizon AP2. Cet obstacle théorique à l'enracinement n'a pas joué, car il est compensé par une plus forte porosité et une moindre compaction; de ce fait, c'est à la base de l'horizon sous-jacent que se marque la différence d'enracinement entre les deux profils. L'horizon $\mathrm{C} 1$ étant identique dans les 2 profils au point de vue de la charge en éléments grossiers, la variation d'enracinement ne peut pas être non plus imputée à cette composante pédologique.

\section{Analyse physico-chimique des sols}

L'analyse de la terre fine est remarquablement comparable dans les 2 cas, ainsi que les mesures de stabilité structurale et de réserve en eau (Tableau II). Un seuil apparaît nettement entre les horizons pédogénisés (AP1, AP2 et (B)) et les horizons géologiques ( $\mathrm{C} 1$ et $\mathrm{C} 2$ ), constitués de calcaire quasi pur. Le test de percolation $(\log 10 \mathrm{k} 1)$ montre nettement la chute brutale de la vitesse de circulation de l'eau en profondeur, confirmée par la plus forte amplitude des variations d'humidité entre les pF 3,5 et 4,2. Cette craie est à l'évidence microporeuse, et les remontées d'eau par capillarité y compensent aisément le déficit hydrique climatique, comme dans la majorité des sols crayeux (Vachier et Dever, 1984). L'alimentation hydrique du blé cultivé dans un tel sol ne devrait donc pas être un facteur limitant du développement et du rendement. Les analyses physiques des deux profils n'expliquent pas, en tout cas, les différences d'enracinement observées au cours de l'étude morphologique des 2 situations, “infesté» et "assaini".

Les analyses chimiques aussi sont tout à fait comparables d'une situation à l'autre. II n'y a pas de facteur limitant, sauf un léger déficit en magnésium dans les horizons labourés, où le rapport $\mathrm{Mg} / \mathrm{K}$ est inférieur à 1 . Ces résultats n'expliquent pas non plus les différences d'enracinement, et l'alimentation chimique des végétaux ne peut pas être considérée comme un facteur limitant de leur développement.

\section{Densités résiduelles de nématodes}

Tous niveaux confondus, le site “infesté» présente une densité significativement plus élevée de kystes résultant de la culture de l'avoine multiplicatrice «Peniarth", pendant les trois années précédentes (Tableau III). Les infestations sont significativement différentes, pour chaque tranche, entre les 2 sites. Dans le site “infesté», l'essentiel de l'infestation est localisé dans les 40 premiers $\mathrm{cm}$, plus particulièrement entre 20 et $40 \mathrm{~cm}$, alors qu'il n'est observé aucun nématode en dessous de $60 \mathrm{~cm}$.

Dans le site "assaini», la densité résiduelle de kystes est significativement la plus élevée entre 40 et $60 \mathrm{~cm}$ de profondeur. II n'y a pas de différence significative entre les 2 tranches $0-20 \mathrm{~cm}$ et $20-40 \mathrm{~cm}$, mais des kystes sont dénombrés dans le niveau le plus profond $60-80$ $\mathrm{cm}$.

La densité de larves viables par kyste présente une distribution analogue à celle des enve- 
Tableau II. Résultats analytiques des horizons pédologiques dans deux profils "assaini» ou «infesté» par Heterodera avenae (Prélèvements du 8 octobre 1986).

$$
A P_{1} \quad A P_{2} \begin{gathered}
\text { "assaini" } \\
(B)
\end{gathered} \quad C_{1} \quad C_{2} \quad A P_{1} \quad A P_{2} \quad \text { (B) } C_{1} \quad C_{2}
$$

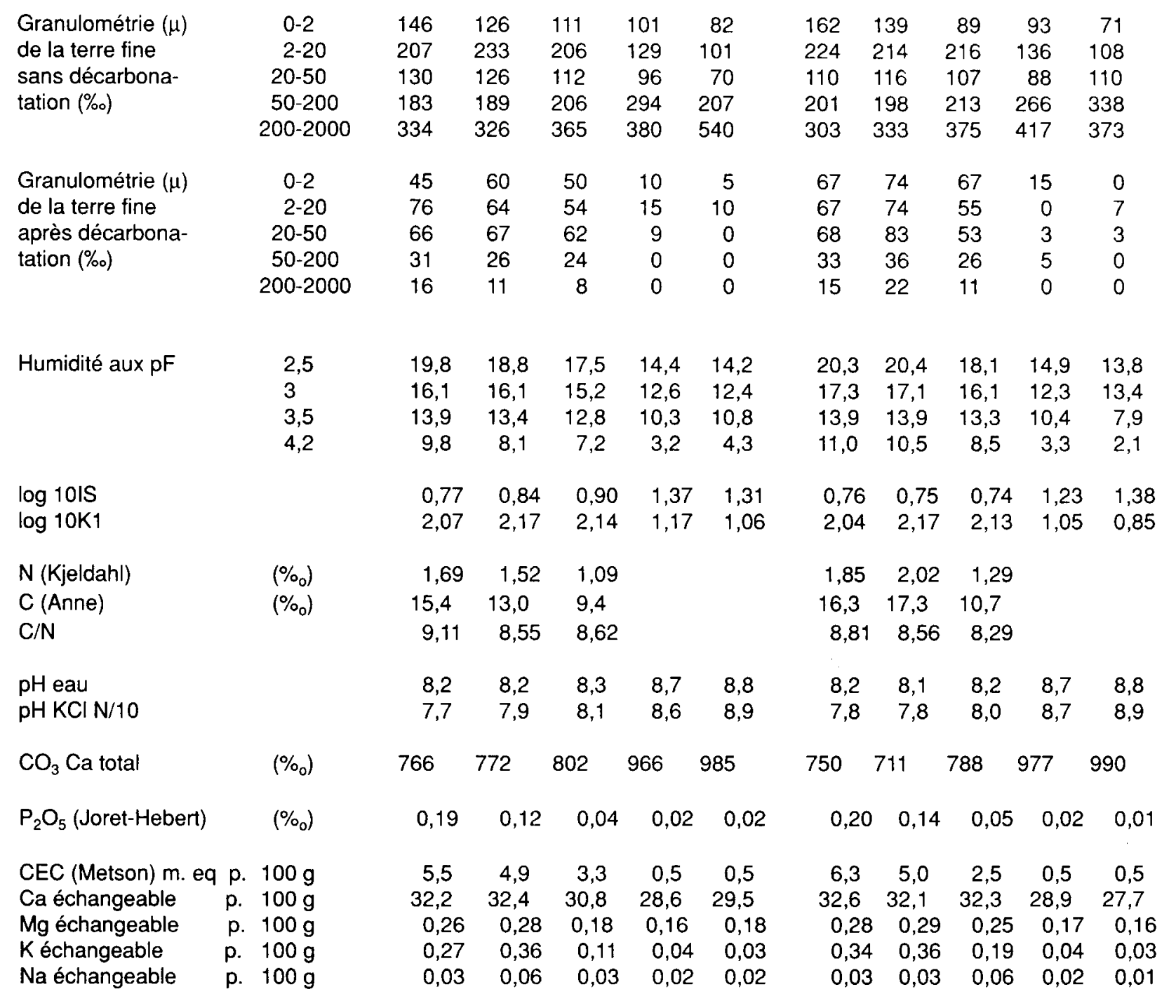

loppes maternelles (Tableau IV). L'infestation résiduelle dans le site «infesté» est aussi essentiellement localisée entre 0 et $40 \mathrm{~cm}$; la deuxième tranche étant néanmoins la plus infestée. II n'est cependant pas observé de larves viables en dessous de $40 \mathrm{~cm}$.

Dans la zone «assainie», l'infestation est logiquement faible, en raison de la culture de la variété résistante «Panema», pendant les 3 années précédentes; mais on observe le taux d'infestation le plus élevé entre 40 et $60 \mathrm{~cm}$ de profondeur (profondeur à laquelle on trouve aussi la densité de kystes la plus élevée).

\section{Distribution des nématodes nouvellement formés}

Compte tenu des densités préculturales, c'est logiquement, dans les 2 premiers niveaux du site "infesté" que la nouvelle génération de nématodes est la plus dense (Tableau V). Les individus les plus nombreux sont entre 0 et $20 \mathrm{~cm}$, où ont été enregistrées les masses de racines les plus importantes (Tableau I). La quasi-absence de multiplication du nématode en dessous de $40 \mathrm{~cm}$ résulte d'une prospection réduite par les racines et d'une densité d'infestation initiale faible ou nulle. 
Tableau III. Densités des kystes anciens d'Heterodera avenae dans différents niveaux de sols "assaini" et «infesté", cultivés avec le blé tendre d'hiver “Fidel»: moyennes des données transformées $(\log (x+1))(1)$ et brutes $(2)$; différences significatives (S) et P.PD.S. $(\Delta)$ à $P=0,05$; les moyennes suivies de la même lettre ne sont pas significativement différentes les unes des autres.

\begin{tabular}{|c|c|c|c|c|c|c|c|}
\hline & \multirow[t]{2}{*}{ Site } & \multicolumn{4}{|c|}{ Protondeur $(\mathrm{cm})$ des niveaux de sols } & \multirow{2}{*}{$\begin{array}{l}\text { Niveaux } \\
\text { confondus }\end{array}$} & \multirow{2}{*}{$\begin{array}{l}\text { Différences } \\
\text { entre niveaux } \\
\text { "assaini/infesté" }\end{array}$} \\
\hline & & $0-20$ & $20-40$ & $40-60$ & $60-80$ & & \\
\hline \multirow{4}{*}{$\begin{array}{l}\text { Kystes/210 ml } \\
\text { de sol prélevé }\end{array}$} & "assaini" & $0,98 b(8,8)$ & $1,02 b(9,6)$ & $1,49 c(30,4)$ & $0,73 a(4,8)$ & $1,06(13,4)$ & $0-20 \mathrm{~S}$ \\
\hline & $(\Delta=0.16)$ & (1) (2) & & & & & $\begin{array}{l}20-40 \mathrm{~S} \\
40.60 \mathrm{~S}\end{array}$ \\
\hline & $\begin{array}{l}\text { «infesté" } \\
(\Delta=0.11)\end{array}$ & $2,06 c(113,8)$ & $2,48 d(302,6)$ & $0,12 b(0,4)$ & oa & $1,17(104,2)$ & $60-80 \mathrm{~S}$ \\
\hline & $\Delta$ (intersite) & & & & & 0,07 & 0,13 \\
\hline \multirow{4}{*}{$\begin{array}{l}\text { Kystes/100 gl } \\
\text { de sol séché }\end{array}$} & $\begin{array}{c}\text { "assaini" } \\
(\Delta=0.14)\end{array}$ & $0,71 b(4,2)$ & $0,69 b(4,0)$ & $1,10 c(11,7)$ & $0,45 a(2,0)$ & $0,74(5,5)$ & $\begin{array}{r}0-20 \mathrm{~S} \\
20-40 \mathrm{~S}\end{array}$ \\
\hline & & & & & & & $40-60 \mathrm{~S}$ \\
\hline & $\begin{array}{c}\text { «infesté»" } \\
(\Delta=0.06)\end{array}$ & $1,73 b(52,9)$ & $2,08 d(119,1)$ & 0,06 a $(0,2)$ & Oa & $0,97(43,1)$ & $60-80 \mathrm{~S}$ \\
\hline & $\Delta$ (intersite) & & & & & 0,05 & 0,01 \\
\hline
\end{tabular}

Tableau IV. Densités résiduelles (larves viables/kyste) d'Heterodera avenae dans différents niveaux de sols "assaini» et “infesté", cultivés avec le blé tendre d'hiver "Fidel»: moyennes des données transformées $(\log (x+1))(1)$ ou brutes (2); différences significatives (S) et P.P.D.S. $(\Delta)$ à $P=0,05$; les moyennes suivies de la même lettre ne sont pas significativement différentes les unes des autres.

\begin{tabular}{|c|c|c|c|c|c|c|}
\hline \multirow[t]{2}{*}{ Site } & \multicolumn{4}{|c|}{ Profondeur (cm) des niveaux de sols } & \multirow{2}{*}{$\begin{array}{l}\text { Niveaux } \\
\text { confondus }\end{array}$} & \multirow{2}{*}{$\begin{array}{l}\text { Différence } \\
\text { entre niveaux } \\
\text { "assaini/infesté»" }\end{array}$} \\
\hline & $0-20$ & $20-40$ & $40-60$ & $60-80$ & & \\
\hline «assaini» & $\begin{array}{c}0,22 \text { a }(2,2) \\
(1) \quad(2)\end{array}$ & $0 a$ & $0,45 a(2,5)$ & $0 a$ & $0,17(1,2)$ & $\begin{array}{r}0-20 \mathrm{~S} \\
20-40 \mathrm{~S} \\
40-60 \mathrm{~S}\end{array}$ \\
\hline $\begin{array}{l}\text { "infesté»" } \\
(\Delta=0.04)\end{array}$ & $1,18 b(14,1)$ & $1,20 b(14,9)$ & $0 a$ & $0 a$ & $0,60(7,3)$ & $60-80$ NS \\
\hline$\Delta$ (intersite) & & & & & 0,15 & 0,29 \\
\hline
\end{tabular}

II y a production d'un nombre réduit de femelles en site «assaini», dans les 3 niveaux compris entre 0 et $60 \mathrm{~cm}$. L'augmentation significative du nombre d'individus entre 40 et $60 \mathrm{~cm}$ conduit à une densité de population qui, par ailleurs, se démarque statistiquement de la densité de la tranche homologue en site «infesté». Cette densité relativement forte s'explique par une présence résiduelle plus élevée du nématode à cette profondeur et par la progression du système radiculaire. Le labour du site «infesté» a pour effet d'enfouir les nématodes nouvellement formés, puisque les densités en larves par $\mathrm{g}$ de sol et par kyste sont significativement plus fortes entre 20 et $40 \mathrm{~cm}$ qu'entre 0 et $20 \mathrm{~cm}$ de profondeur (Tableau VI).

\section{DISCUSSION-CONCLUSION}

Les propriétés physiques du sol exercent une grande influence sur le développement du système radiculaire, notamment par la présence d'horizons instables et compacts, qui freinent la colonisation du sous-sol par les racines (Maertens, 1964). Les différences dans la structure pédologique de ces 2 sites n'expliquent pas une telle opposition à l'enracinement dans la zone infestée 
par $H$. avenae. Le nématode est donc seul responsable des modifications de la croissance et de l'extension du système radiculaire. L'augmentation de la masse de racines de céréales infestées par $H$. avenae est un phénomène fréquemment observé (Andersen, 1961; Williams et Salt, 1970; Rivoal et Sarr, 1987). Elle est due à la croissance de nombreuses racines latérales du système séminal, qui peut être éventuellement confondue avec des réactions de la plante à des contraintes mécaniques (Price et al., 1983).

Les cultures peuvent manquer d'eau si leurs systèmes radiculaires ne pénètrent pas dans les couches profondes du sol. La colonisation insuffisante du sous-sol par les racines dans le site «infesté» est vraisemblablement préjudiciable à la culture de blé, lorsque les disponibilités hydriques diminuent en surface, en période de sécheresse (Ballif, 1981). Maertens (1964), Maertens et al. (1974) ainsi que Blanchet et al. (1974) ont démontré le rôle prédominant des racines profondes dans l'absorption de l'eau, des phosphates et de l'azote minéral, lors de l'assèchement de l'horizon cultivé.

Ces différences d'enracinement expliquent également la variabilité dans la distribution en profondeur des nématodes, aussi bien pour les populations résiduelles que pour les individus nouvellement formés. A cause de l'arrêt de l'enracinement à $40 \mathrm{~cm}$ de profondeur en zone

Tableau V. Densités de femelles blanches et kystes nouveaux d'Heterodera avenae dans le profil pédologique des sites "assaini" et "infesté" cultivés avec le blé tendre d'hiver "Fidel». Moyennes des données transformées (log $(x+$ 1)) (1) ou brutes ((2); différences significatives (S) et P.P.D.S. $(\Delta)$ à $P=0,05$; les moyennes suivies de la même lettre ne sont pas significativement différentes les unes des autres.

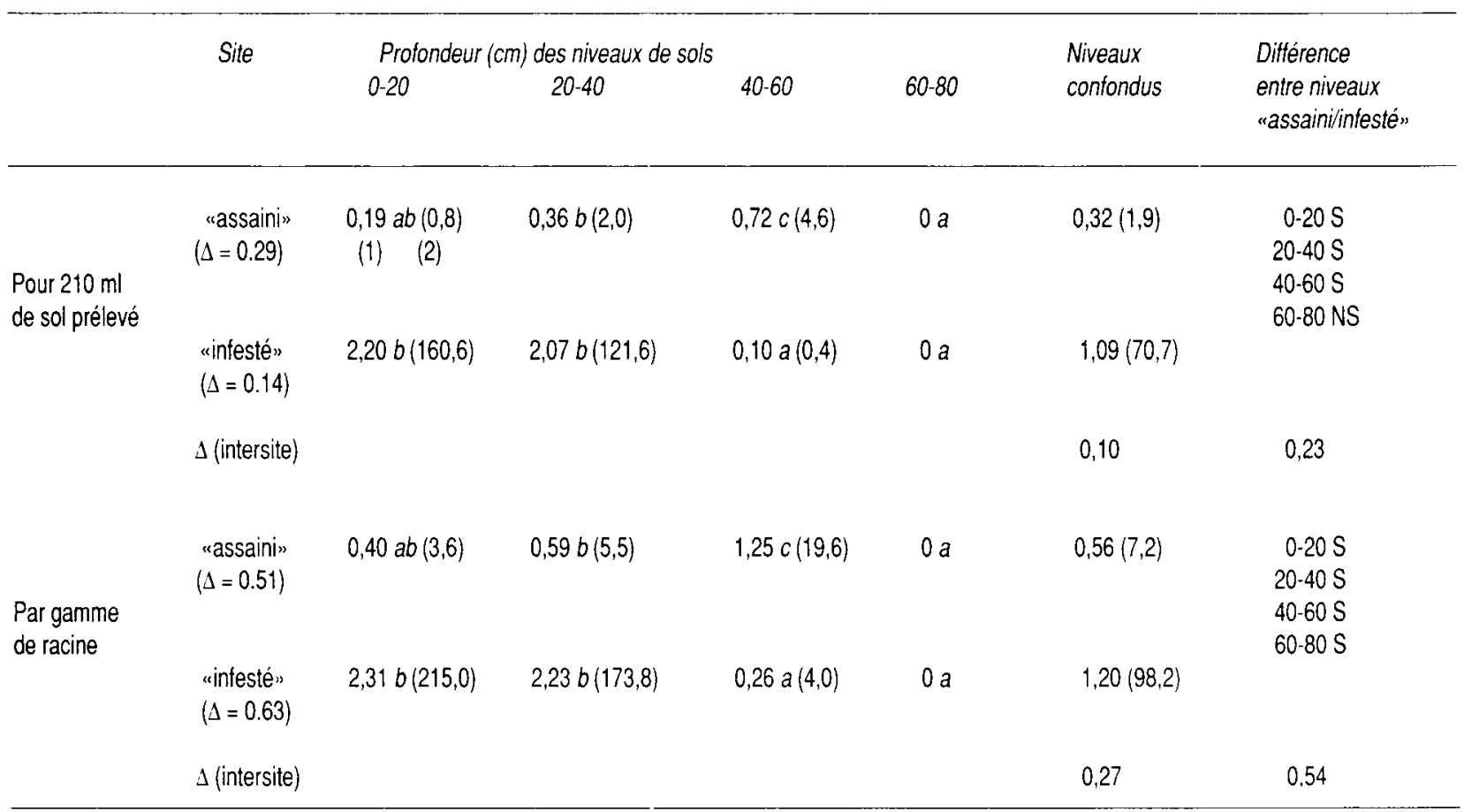

Tableau VI. Densités d'Heterodera avenae dans le sol cultivé avec le blé tendre d'hiver "Fidel», après labour: moyennes des données transformées $(\log (x+1))(1)$ ou brutes $(2)$ et signification du test de Fisher à $P=0,05$.

\section{Profondeur $(\mathrm{cm})$ des prélèvements Test $F$}

$0-20 \quad 20-40$

$\begin{array}{llll}\text { Kystes/100 g de sol séché } & 1,87(1)(72,0)(2) & 1,88(78,4) & 0,04 \mathrm{NS} \\ \text { Larves/g sol séché } & 1,17(13,8) & 1,36(22,1) & 30,89 \mathrm{~S} \\ \text { Larves/kystes } & 1,57(36,8) & 1,71(50,6) & 13,54 \mathrm{~S}\end{array}$


«infestée», il y a concentration des nématodes dans la couche arable. L'analyse nématologique, après labour du site "infesté", montre que, dans les études de dynamique des populations, l'échantillonnage effectué après une culture hôte sur une profondeur de 15 à $20 \mathrm{~cm}$ (Rivoal et al., 1986; Rivoal et Sarr, 1987) concerne des effectifs moins importants que ceux de l'horizon sousjacent, $20-40 \mathrm{~cm}$. Dans un sol argilo-humifère, Whitehead et al. (1979) ont également constaté pour $H$. schachtii, après betterave, des densités plus élevées entre 20 et $60 \mathrm{~cm}$ qu'entre 0 et 20 $\mathrm{cm}$ de profondeur. Le niveau $20-40 \mathrm{~cm}$, partiellement remis en surface par le labour, héberge une population âgée d'au moins 1 année, dont la densité a chuté par éclosion naturelle des larves (Kerry et Jenkinson, 1976; Rivoal, 1986a).

L'échantillonnage de la couche superficielle du sol fait sous-estimer les densités larvaires avant la mise en place de la culture. Le potentiel infectieux enfoui entre 20 et $40 \mathrm{~cm}$ constitue, en outre, une véritable barrière biologique pour l'exploration des couches profondes du sol par le système radiculaire de la culture consécutive de céréale hôte.

Le recouvrement de kystes dans le profil "assaini" confirme la pérennité de ces enveloppes maternelles, qui contiennent les larves infectieuses (Shepherd, 1962). Celles-ci sont peu nombreuses, en raison de l'absence de plantes multiplicatrices pendant les 3 années précédentes et de la forte propension des larves à éclore chez les populations septentrionales de $H$. avenae (Rivoal, 1983; Rivoal et Sarr, 1983). La plus forte densité de nématodes enregistrée entre 40 et $60 \mathrm{~cm}$ de profondeur, aussi bien en densités résiduelles qu'en effectifs nouvellement formés, est inattendue. Elle pourrait résulter d'un déplacement d'individus endoparasites provenant de niveaux supérieurs, du fait de la croissance en profondeur des racines. Une autre hypothèse est la descente par gravité, éventuellement accentuée par la circulation de l'eau pluviale, de nématodes libres empruntant l'importante porosité du sol.

Le protocole expérimental mis en place à Argentan offre de réelles possibilités pour évaluer, au champ, l'impact de $H$. avenae sur l'écophysiologie du blé tendre d'hiver. Les résultats obtenus feront l'objet d'une prochaine publication. Cette procédure paraît également appropriée pour l'étude des conséquences d'attaques par un agent pathogène tellurique sur les modalités d'alimentation hydrique et minérale des plantes (Maertens et al., 1987). Les données sur ce sujet sont fragmentaires, peu comparables entre elles, et partois contradictoires (Ritter, 1976). Ainsi, Trudgill et al. (1975) expliquent que l'inhibition de la croissance de la pomme de terre infestée par Globodera résulte d'un défaut d'assimilation du potassium. Par contre, Price et al. (1982) constatent que les attaques de $H$. avenae sur avoine n'empêchent pas l'assimilation du potassium ni du phosphore, du fait de l'augmentation de la capacité d'absorption des racines. L'usage de traceurs isotopiques pourrait accroître les potentialités de ce type d'investigations (Price et Sanderson, 1984).

\section{RÉFÉRENCES}

Andersen S. (1961). Resistens mod havreal. Dissertation. Danskidenskabs Forlag. Kobenhavn.

Ballif J.L. (1981) Comportement hydrique d'une rendzine brune sur craie sous l'influence de la sécheresse. C.R. Acad. Agric. Fr. 67, 395-403

Blanchet R., Bosc M., Maertens C. \& Marty J.R. (1974) Influence de différents régimes hydriques sur l'absorption de l'eau et des éléments minéraux par les cultures. III. Alimentation minérale des plantes en culture irriguée ou non, et répercussions sur la fertilisation. Ann. Agron. 25, 821-836

Doussinault G., Person-Dedryver F., Dosba F. \& Rivoal R. (1986) La sélection de blés tendres résistants au développement de Heterodera avenae. In: "Les rotations céréalières intensives". Dix années d'études concertées INRA-ONIC-ITCF, 1973-1983. INRA Paris, 203-213

Henin S., Gras S. \& Monnier G. (1969) Le Profil Cultural. Masson, $2^{\mathrm{e}}$ ed.

Hervé Y. (1982) Expérimentation Agronomique: Tech nique des Essais Agricoles. Document ENSA

Kerry B.R. \& Jenkinson S.C. (1976) Observations on emergence, survival and root invasion of second stage larvae of the cereal cyst nematode, Heterodera avenae. Nematologica 22, 467-474

Lucas P., Lemaire J.M., Doussinault G., Perraton B., Tivoli B. \& Carpentier F. (1986) Lutte biologique contre Gaeumannomyces graminis (Sacc.) Arx et Olivier var. tritici (Walker), agent du piétin-échaudage, par l'utilisation d'une souche hypo-agressive du parasite. Résultats, perspectives. In: "Les rotations céréalières intensives". Dix années d'études concertées INRAONIC-ITCF, 1973-1983. INRA Paris, 113-125

Maertens C. (1964) Influence des propriétés physiques des sols sur le développement radiculaire et conséquences sur l'alimentation hydrique et azotée des cultures. Science du Sol 2, 1-11

Maertens C., Blanchet R. \& Puech J. (1974) Influence de différents régimes hydriques sur l'absorption de l'eau et des éléments minéraux par les cultures. 1. - Régimes hydriques, systèmes racinaires et modalités d'alimentation en eau. Ann. Agron., 25, 575-586

Maertens C., Masse J., Picard D. \& Tardieu F. (1987) Mise en place et fonctionnement du système racinaire des principales espèces cultivées: recherches en cours à l'INRA et à l'ITCF. Perspect. Agric. 119, 6-11

Meagher J.W. (1977) World dissemination of the cereal-cyst nematode (Heterodera avenae) and its potential as a pathogen of wheat. J. Nematol. 9, 9-15

Price N.S. \& Sanderson J. (1984) The translocation of calcium from oat roots infested by the cereal cyst 
nematode Heterodera avenae Woll. Rev. Nématol. 7 , 239-243

Price N.S., Clarkson D.T. \& Hague N.G.M. (1982) The uptake of potassium and phosphorus in oats infested with the cereal cyst nematode, Heterodera avenae Woll. Rev. Nematol. 5, 321-325

Price N.S., Clarkson D.T. \& Hague N.G.M. (1983) Effect of invasion by cereal cyst nematode (Heterodera avenae) on the growth and development of the seminal roots of oats and barley. Plant Pathol. 32, 377383

Ritter M. (1976) The interaction between nutrients and host resistance to nematodes with reference to mediterranean crops. Proc. 12th Coll. Intern. Potash Inst. Izmir, Turkey, 291-299

Ritter M. (1982) Importance des nématodes à kystes des céréales, Bull. OEPP, 12, 307-316

Rivoal R. (1973) Etude de la nuisibilité du nématode à kystes des racines de céréales (Heterodera avenae Woll.) en France et des causes de sa variabilité. C.R. Acad. Agric. 12, 959-970

Rivoal R. (1982) Caractérisation de deux écotypes d'Heterodera avenae en France par leurs cycles et conditions thermiques d'éclosion. Bull. OEPP. 12, 353359

Rivoal R. (1983) Biologie d'Heterodera avenae Wollenweber en France. III. Evolution des diapauses des races $\mathrm{Fr} 1$ et $\mathrm{Fr} 4$ au cours de plusieurs années consécutives; influence de la température. Rev. Nématol. 6 , 157-164

Rivoal R. (1986a) Biology of Heterodera avenae Wollenweber in France. IV. Comparative study of the hatching cycles of two ecotypes after their transfer to different climatic conditions. Rev. Nématol. 9, 405-410

Rivoal R. (1986b) Nématodes: influence d'Heterodera avenae. Agro-maïs. 46, 18-20

Rivoal R. \& Sarr E. (1983) Considérations sur l'emploi éventuel de nématicides endothérapiques ou à action de contact, appliqués à faibles doses, pour lutter contre le nématode à kyste des céréales, Heterodera avenae, en France. Phytiatr. Phytopharm. 32, 177-186 Rivoal R. \& Sarr E. (1987) Field experiments on Heterodera avenae in France and implications for winter wheat performance. Nematologica. 33, 460-479

Rivoal R., Person F., Caubel G. \& Scotto La Massese C. (1978) Méthodes d'évaluation de la résistance des céréales au développement des nématodes: Ditylenchus dipsaci, Heterodera avenae et Pratylenchus spp. Ann. Amélior. Plant. 28, 371-394

Rivoal R., Besse T., Morlet G. \& Penard P. (1986) Les Rotations Céréalières Intensives. Nuisibilité du Nématode à Kyste Heterodera avenae et Perspectives de Lutte. Ouvr. INRA. 153-173

Shepherd A.M. (1982) The emergence of larvae from cysts in the genus Heterodera. Techn. Comm. Number. 32. Comm. Bur. Helm. St-Albans. Herts. England

Trudgill D.L., Evans K. \& Parrott D.M. (1975) Effects of potato cyst-nematodes on potato plants. I. Effects in a trial with irrigation and fumigation on the growth and nitrogen and potassium contents of a resistant and susceptible variety. Nematologica 21, 169-182

Turner E.M. (1953) The nature of the resistance of oats to the take of all fungus. J. Exp. Bot. 4, 264-271

Vachier P. \& Dever L. (1984) Isotopic approach of water movement in the chalk insaturated zone. Proc. Symp. Recent investigations in the zone of aeration. Munich. 1, 37-46

Whitehead A.G., Tite D.J., Finch P.H., Fraser J.E. \& French E.M. (1979) Chemical control of beet cystnematode, Heterodera schachtii, in some peaty loam soils. Ann. Appli. Biol. 92, 73-79

Williams T.D. \& Salt G.A. (1970) The effects of soil sterilants on the cereal cyst nematode (Heterodera avenae Woll.), take-all (Ophiobulus graminis Sacc.) and yields of spring wheat and barley. Ann. Appl. Biol., 66, 329-338 